\title{
One-pot green synthesis of copper(I) iodide nanocrystallites using red cabbage extract and its application for the colorimetric sensing of elemental mercury vapor
}

\author{
Alan Rodelle M. Salcedo ${ }^{1 *} \mathcal{E}$ Fortunato Sevilla III ${ }^{1,2}$ \\ ${ }^{1}$ The Graduate School; ${ }^{2}$ College of Science; ${ }^{3}$ Research Center for the Natural and Applied Sciences, \\ University of Santo Tomas, 1015 Manila, Philippines
}

\begin{abstract}
CuI nanocrystallites were synthesized via a red cabbage-mediated one-pot method at ambient temperature. Red cabbage extract functioned as reducing agent, converting $\mathrm{Cu}(\mathrm{II})$ into $\mathrm{Cu}(\mathrm{I})$ and as a capping agent stabilizing the $\mathrm{CuI}$ nanocrystallites. The resulting material was characterized using X-ray powder diffraction, scanning electron microscopy with energy dispersive $\mathrm{X}$-ray spectroscopy, differential scanning calorimetry, thermal gravimetric analysis, and UV-Vis spectroscopy. It was also characterized as a solid-state colorimetric sensing reagent for elemental $\mathrm{Hg}^{0}$ vapor through a smartphone camera-based digital image analysis. The sensor exhibited a colorimetric response to $\mathrm{Hg}^{0}$ vapor at a concentration range of 11.4$32.6 \mathrm{ppb}_{\mathrm{v}} \mathrm{Hg}^{0}$ with detection limit of $3.9 \mathrm{ppb}_{\mathrm{v}} \mathrm{Hg}^{0}$, indicating the capability of the sensor to detect the current threshold exposure limit set by the World Health Organization $\left(6.1 \mathrm{ppb}_{\mathrm{v}}\right)$. The sensor performance characteristics confirm that the green-synthesized $\mathrm{CuI}$ can be a suitable colorimetric sensitive layer for detection of $\mathrm{Hg}^{0}$ vapor.
\end{abstract}

Keywords: green chemistry, cuprous iodide, smartphone camera, colorimetric sensor, mercury vapor

\section{INTRODUCTION}

Copper(I) iodide (CuI) has been recognized as a versatile functional material, finding applications in diverse fields, including organic electronics, optoelectronics, semiconductors, and transparent thermoelectrics [1-3]. It is also an efficient catalyst in organic synthesis [4-6], and a potential protective agent against bacterial and viral infections $[7,8]$. It has likewise been

*To whom correspondence should be addressed: amsalcedo@ust.edu.ph employed as a solid-state chromogenic sensing material for $\mathrm{Hg}^{0}$ vapor $[9,10]$.

CuI has been prepared through two basic chemical routes: iodination and precipitation reaction. Iodination is a gas-solid phase reaction, involving iodine vapor and a thin film of copper or a copper salt $[11,12]$. Precipitation is usually conducted in a solution medium containing copper salt and potassium iodide, together with a reducing agent and a stabilizing agent $[13,14]$. These methods are coupled with physical methods to generate nanostructured CuI. 
In order to make the synthesis of CuI costeffective and environmental-friendly, green strategies were applied. A solvent-free approach involved a mechanochemical technique applied in an all solid state precipitation reaction [15]. Green reagents, such as plant or fruit extracts, were used as the reducing agent and the stabilizing agent in the precipitation method. Among the reagents used in the green synthesis of copper(I) iodide were sugar beet juice [16], pomegranate juice [17] and kidney bean extract [7]. Plant-derived reagents have provided green alternatives in the synthesis of nanomaterials [18].

In this study, we report a green synthesis of CuI via a red cabbage extract-mediated one-pot method. The synthesized nanocrystalline CuI was characterized and explored as a colorimetric reagent for the sensing of mercury vapor $\left(\mathrm{Hg}^{0}\right)$. The sensing reaction involved the formation of the red-orange cupro-tetraiodo-mercuriate complex when $\mathrm{Hg}$ reacts with CuI [19] according to Equation (1):

$$
4 \mathrm{CuI}+\mathrm{Hg}^{0} \rightarrow \mathrm{Cu}_{2}\left[\mathrm{HgI}_{4}\right]+2 \mathrm{Cu}^{0}
$$

This color reaction has been previously applied to detect $\mathrm{Hg}$ vapor in a gas mixture, such as air [9] or Hg vapor generated from mercury species in samples such as fish, soil, sediment and gold mining residues [10]. Color measurement was realized using digital imaging devices, such as the smartphone camera. The measurement of elemental mercury vapor is of particular importance due to the inherent toxic effect of mercury to human health and the environment.

\section{EXPERIMENTAL SECTION}

One-pot synthesis. Reagent-grade $\mathrm{CuSO}_{4}$ and KI were procured from Sigma-Aldrich and used without further purification. $\mathrm{CuSO}_{4}$ solution $(0.12 \mathrm{M})$ was slowly mixed under magnetic stirring with red cabbage extract which was prepared by boiling $100 \mathrm{~g}$ of cut red cabbage in $200 \mathrm{~mL}$ water. KI solution (0.12 M) was then added dropwise and the mixture was continuously stirred for 30 min under ambient temperature. The resulting dark gray precipitate was filtered and washed repeatedly with ultrapure water and ethanol to remove impurities. The CuI precipitate was dried in an oven at $50^{\circ} \mathrm{C}$. This procedure was carried out using different volumes of red cabbage extract: 1.0, 5.0, 10.0, and $20.0 \mathrm{~mL}$.

Characterization. The synthesized CuI was characterized using a X-ray powder diffraction (XRD) analyzer (Maxima XRD-7000, Shimadzu), a scanning electron microscope (SEM) with energy dispersive X-ray spectroscopy analyzer (EDS) (JSM-5300, JEOL), a differential scanning calorimeter (DSC) (DSC 4000, Perkin Elmer), a thermal gravimetric analyzer (TGA) (TGA 4000, Perkin Elmer), and UV-Vis spectrophotometer (Lambda 35, Perkin Elmer).

Colorimetric sensing of $\mathbf{H g}^{\mathbf{0}}$. The sensing characteristics of the synthesized $\mathrm{CuI}$ was evaluated using the procedures in a previous publication [9]. $\mathrm{Hg}^{0}$ colorimetric paper sensor was prepared by mixing the synthesized $\mathrm{CuI}$ with a solution of polystyrene (PS, average MW 280,000, Sigma-Aldrich) in tetrahydrofuran (THF, RCI Labscan). The resultant emulsion was applied evenly onto a chromatography paper (Whatman, 3MM CHR) using a rolling glass rod. It was then dried at $40^{\circ} \mathrm{C}$ in an oven for $30 \mathrm{~min}$ to yield a CuI/PS sensing composite on a cellulosic substrate. It was then cut into square pieces $\left(10 \times 10 \mathrm{~mm}^{2}\right)$ and set onto a glass slide for easier handling during the experiments.

The colorimetric paper sensor was placed in a glass vial, and different volumes of standard Hg-air mixture was injected into the vial. The standard gas mixture was generated using the "bell-jar" method [20, 21]. This procedure produced in the vial a gas phase with $\mathrm{Hg}^{0}$ vapor concentrations ranging from 3.4 to $104 \mathrm{ppb}_{\mathrm{v}}$. The actual $\mathrm{Hg}^{0}$ concentration in the vapor phase was determined using Lumex RA915M Mercury Analyzer. 
The intensity of the color developed in the paper sensor was measured by capturing the digital images of the sensor using a smartphone camera (16 megapixels, Samsung S6 Edge) in a light box set up [9]. The acquired images of the paper sensors were digitized to RGB (red-greenblue) color values in a computer using ImageJ $1.49 \mathrm{~h}$ software (National Institute of Health, USA). The sensor response was calculated as percent change $(\% \Delta)$ in the $\mathrm{R}, \mathrm{G}$, or $\mathrm{B}$ values before and after exposure to $\mathrm{Hg}^{0}$ of the paper sensors where $\mathrm{X}$ is the mean color value corresponding to $\mathrm{R}, \mathrm{G}$ or $\mathrm{B}$ (Equation 2).

$$
\% \Delta \mathrm{X}=\frac{\mathrm{X}_{\text {before exposure }}-\mathrm{X}_{\text {after exposure }}}{\mathrm{X}_{\text {before exposure }}} * 100
$$

\section{ResUlts AND DISCUSSION}

Characterization of green-synthesized CuI nanocrystallites. The formation of $\mathrm{CuI}$ was confirmed by the X-ray diffraction pattern obtained from the synthesized product (Fig. 1). The XRD pattern corresponded to a facecentered cubic (fcc) structure of $\gamma$-CuI (JCPDS no. 06-0246, space group: F-43m) [2, 3, 15]. The absence of diffraction peaks due to impurities such as $\mathrm{Cu}$ or $\mathrm{Cu}$ oxides proved the high phase purity of the synthesized material. The average crystallite size calculated using Scherer equation

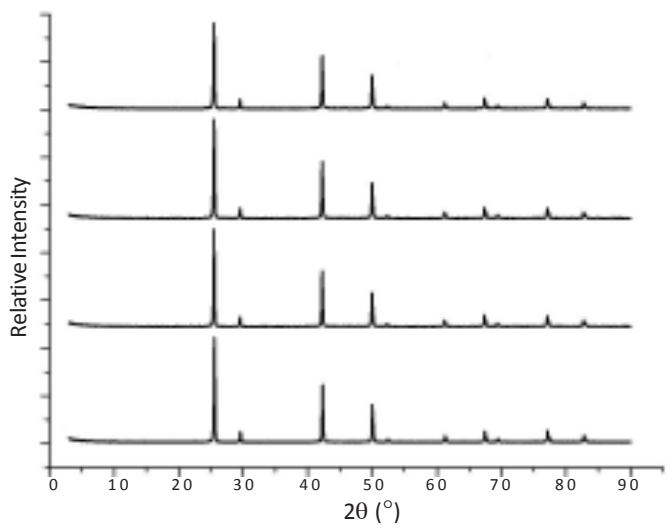

Figure 1. XRD pattern of the CuI product synthesized with 1.0, 5.0, 10.0, and $20.0 \mathrm{~mL}$ of red cabbage extract.
[1] ranged from $85 \mathrm{~nm}$ to $92 \mathrm{~nm}$, depending on the volume of the plant extract used. The CuI nanocrystallites obtained using $5.0 \mathrm{~mL}$ red cabbage extract had the largest size at $92 \mathrm{~nm}$, while those obtained from 10.0 and $20.0 \mathrm{~mL}$ had the smallest size at $85 \mathrm{~nm}$.

The SEM images of the synthesized products revealed that the $\mathrm{CuI}$ powder is made up mostly of triangular micro- and nanostructures that are clustered and agglomerated as shown in Fig. 2. These triangular structures were smaller in size when greater amount of red cabbage extract used during its synthesis. The EDS elemental analysis (Fig. 3) verified the presence of $\mathrm{Cu}$ and I as the major constituents in the synthesized products. It also revealed the presence of $\mathrm{C}$ and $\mathrm{O}$ where the atomic percentage increased as more red cabbage extract was employed in the synthesis. The identified traces of $\mathrm{C}$ and $\mathrm{O}$ could be due to the anthocyanin present in the red cabbage extract acting as capping agents for the $\mathrm{CuI}$ structures. Red cabbage is known to be rich in anthocyanins that can both act as a reducing agent and capping agent in the formation of $\mathrm{CuI}$ $[3,16]$. Figure 4 illustrates the possible interaction of the anthocyanin molecules with $\mathrm{CuI}$ particles. A probable mechanism of $\mathrm{CuI}$ micro/nanostructures formation can be

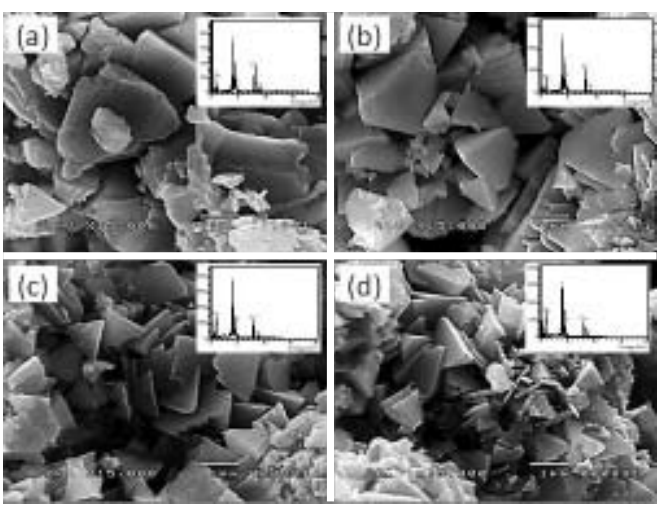

Figure 2. SEM images of the CuI products synthesized using (a) 1 , (b) 5 , (c) 10 , and (d) $20 \mathrm{~mL}$ of red cabbage extract. Inset plots are the respective EDS profile of CuI products (Magnification is $15 \mathrm{kX}$ ). 


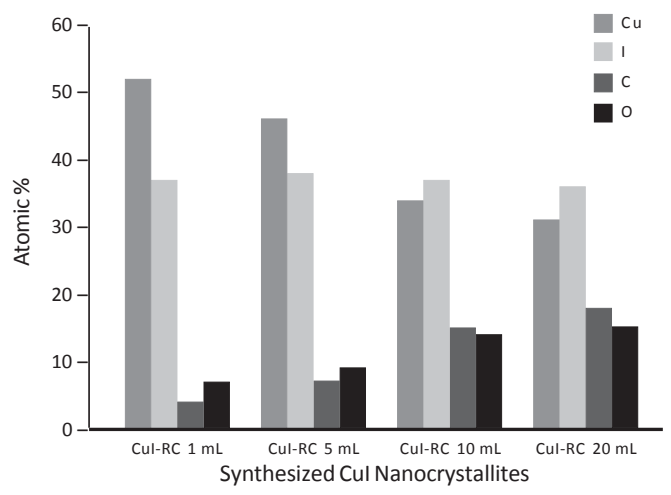

Figure 3. Summary of EDS analysis profile showing atomic percentage of $\mathrm{Cu}, \mathrm{I}, \mathrm{C}$, and $\mathrm{O}$ in the synthesized products using different volumes of red cabbage extract.

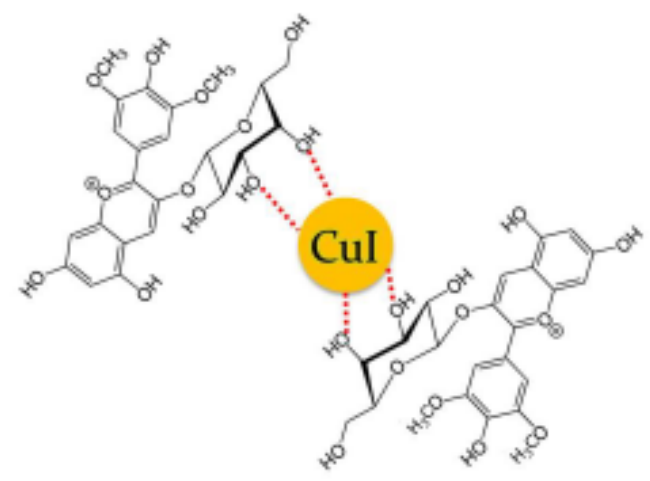

Figure 4. Illustration of the capping agent role of anthocyanin in CuI crystallite.

comparable to the proposed mechanism with glucose [22] since anthocyanin molecules contain glucose subunit [23].

The synthesized $\mathrm{CuI}$ nanocrystallites exhibited UV-Vis absorbance spectra which featured an absorption peaks at $\sim 227 \mathrm{~nm}$ (Fig. 5). No significant difference in the absorption peak wavelength was seen among the spectra of the products which supports the comparable crystallite sizes computed from the XRD data. The absorption peak of nanoparticles is known to be influenced by the particle size, occurring at a lower wavelength for smaller particles [1]. The observed absorption maxima occurred at a

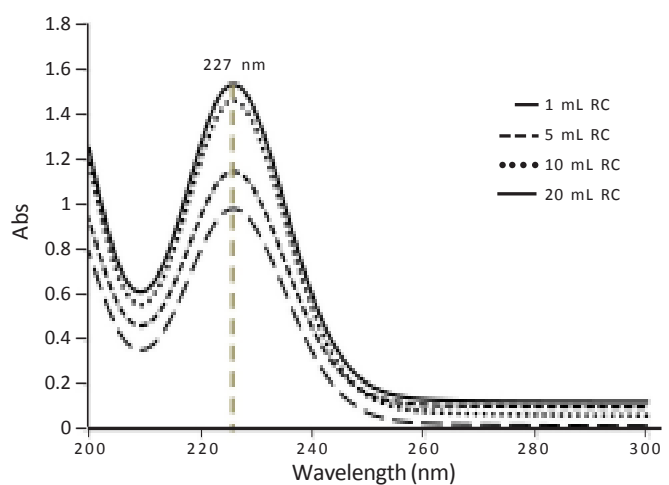

Figure 5. UV-vis spectrum of the CuI nanocrystallites synthesized with $1,5,10$, and and $20 \mathrm{~mL}$ of red cabbage extract.

wavelength higher than that observed from $\mathrm{CuI}$ synthesized from pomegranate juice ( $210 \mathrm{~nm}$ ), wherein smaller particles (35-50 nm) were formed [3]. It is much lower than the peak wavelength observed with bulk CuI (400 nm) [3, 16]. The absorbance of the synthesized CuI was found to increase when the amount of the red cabbage extract was increased. This behavior can be attributed to an increase in the amount of the synthesized CuI products solubilized due to the capping agent.

The DSC curves (Fig. 6A) of the synthesized $\mathrm{CuI}$ nanocrystallites displayed two distinct sharp peaks centered at $\sim 380^{\circ} \mathrm{C}$ and $\sim 403^{\circ} \mathrm{C}$ which can be assigned for the phase transition of $\gamma$-CuI to $\beta$-CuI and $\beta$-CuI to $\alpha$-CuI phases, respectively $[1,13,24]$. These DSC peaks of the synthesized CuI products referring to phase transitions are comparable to that obtained from the bulk CuI [13, 25]. TGA curves of the synthesized CuI (Fig. 6B) showed major weight loss occurring beyond $568-592{ }^{\circ} \mathrm{C}$, which corresponds to the melting temperature of $\mathrm{CuI}$ [26]. These major weight loss zones which extend up to $745-776^{\circ} \mathrm{C}$ can be associated with the loss of iodine in the samples $[1,12]$.

$\mathrm{Hg}^{0}$ vapor-sensing performance of $\mathrm{CuI}$ nanocrystallites. The colorimetric sensing reaction for gaseous elemental mercury using 

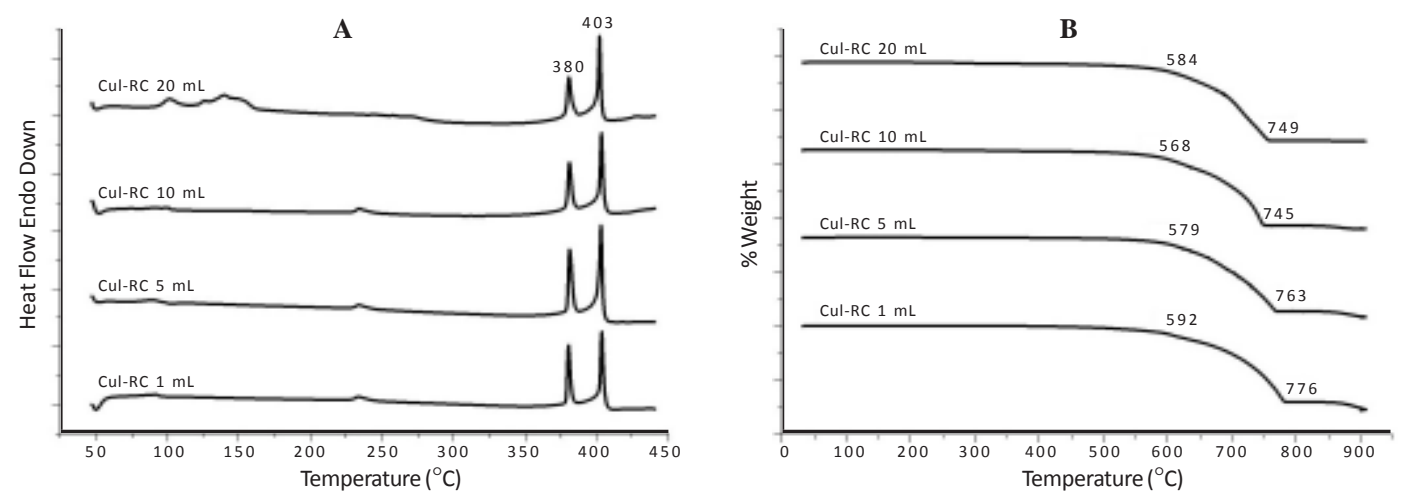

Figure 6. DSC (A) and TGA (B) curves of the synthesized CuI with different volumes of red cabbage extract.

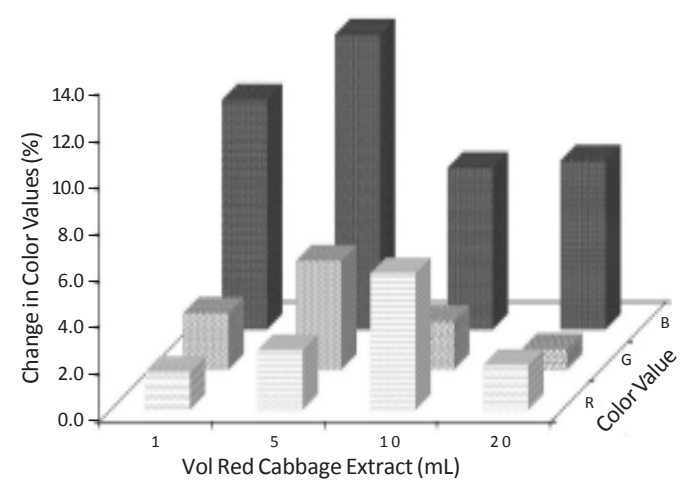

Figure 7. Sensor response in percent change RGB values for the synthesized CuI nanocrystallites with different volumes of red cabbage extract.

$\mathrm{CuI}$ can be conducted only in the gas-solid phase, since the reactants are not soluble in the common aqueous medium. Following our previous work on colorimetric sensing of $\mathrm{Hg}^{0}$ vapor [9], the green-synthesized CuI nanocrystallites was immobilized using a polystyrene binder and supported on a cellulosic substrate. Polystyrene has been proven to be an effective binder for CuI-based colorimetric paper sensor, showing the highest sensor response compared with other polymer binders.

When exposed to $\mathrm{Hg}^{0}$ vapor, the immobilized CuI nanocrystallites exhibited a color variation from very pale gray to red-orange. This color change was measured using digital image colorimetry based on a smartphone camera. The

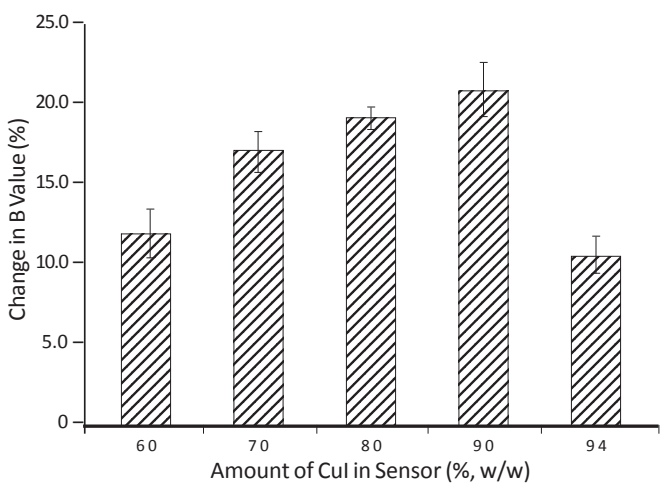

Figure 8. Effect of the amount of $\mathrm{CuI}$ in the colorimetric paper sensing reagent phase on the change in blue response to $45 \mathrm{ppb}_{\mathrm{v}} \mathrm{Hg}^{0}$ vapor. Standard error bars indicate three replicate.

digital image captured by the camera was analyzed in the RGB color space. The exposure of the paper sensor to $\mathrm{Hg}^{0}$ vapor produced distinction in the color values of R, G, and B channels generated from digital image analysis using Image J. The greatest sensitivity to the color change was observed in the $B$ value (Fig. 7) which was consequently used as the sensor response. The color change was found to vary with the amount of red cabbage extract used in the synthesis of CuI. Highest sensor response (in the $\mathrm{B}$ value scale) was observed with the sensor prepared from CuI nanocrystallites synthesized with $5 \mathrm{~mL}$ red cabbage extract. 


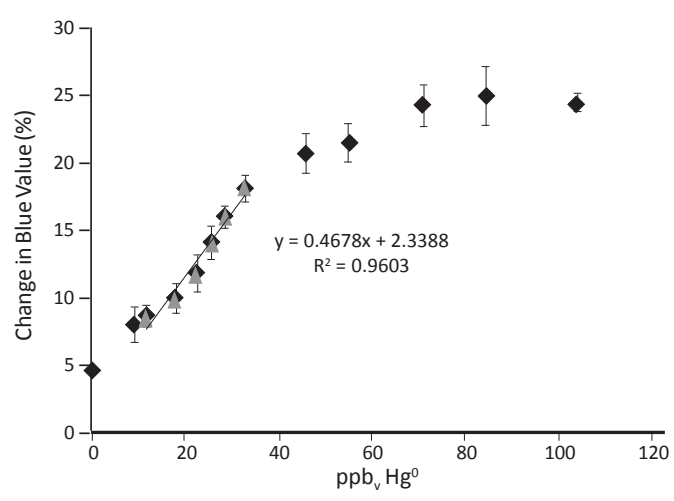

Figure 9. Change in the blue-based response of $\mathrm{CuI}$ nanocrystallites-based colorimetric sensor as a function of the mercury vapor concentration.

Recovery tests on $\mathrm{Hg}^{0}$ in air samples $(n=5)$

\begin{tabular}{c|c|c|c}
\hline Sample & $\begin{array}{c}\text { Added } \\
\text { concentration } \\
\left.\mathbf{( p p b}_{\mathbf{v}} \mathbf{H g}^{\mathbf{0}}\right)\end{array}$ & $\begin{array}{c}\text { Recovered } \\
\text { concentration } \\
\mathbf{( p p b}_{\mathbf{v}} \mathbf{H g}^{\mathbf{0}}, \\
\text { mean } \pm \text { S.D.) }\end{array}$ & $\begin{array}{c}\text { Mean } \\
\text { percent } \\
\text { recovery }\end{array}$ \\
\hline 1 & 20.8 & $21.4 \pm 1.8$ & $103 \pm 8.6$ \\
2 & 22.9 & $22.6 \pm 1.4$ & $99 \pm 6.0$ \\
3 & 30.8 & $31.1 \pm 1.1$ & $101 \pm 4.2$ \\
\hline
\end{tabular}

The sensor response was also dependent on the percentage of $\mathrm{CuI}$ in the sensing reagent phase. The maximum sensitivity was obtained from a 90\% CuI composition (w/w, CuI:polystyrene) (Fig. 8). The high concentration of CuI nanocrystallites in the reagent phase favored the formation of a colored $\mathrm{Cu}_{2}\left[\mathrm{HgI}_{4}\right]$ complex subsequently producing high color intensity in the colorimetric sensing paper. The observed decrease in the sensor response at $94 \% \mathrm{CuI}$ was due to the unstable sensing phase which cracked upon drying.

The sensor response varied with concentration of $\mathrm{Hg}^{0}$ vapor. As seen in Fig. 9, the best linear behavior $\left(r^{2}=0.9603\right)$ was established in the concentration range of $11.4-32.6 \mathrm{ppb}_{\mathrm{v}} \mathrm{Hg}^{0}$ vapor. Beyond this range, there was only a small variation in the response which caused a lower sensitivity. The sensor exhibited very good reproducibility, showing a relative standard deviation ranging from $5.1 \%$ to $10.0 \%$. The limit of detection was calculated based on $3 \mathrm{sd}_{\text {blank }} /$ slope to be $3.9 \mathrm{ppb}_{\mathrm{v}} \mathrm{Hg}^{0}$.

The sensor exhibited a highly satisfactory analytical performance in recovery tests using spiked air samples (Table). The performance of the sensor indicates the promising sensor application for the green-synthesized CuI, particularly for an environmentally importance such as $\mathrm{Hg}^{0}$ vapor.

\section{Conclusion}

Triangular nanocyrstallites of $\gamma$-CuI was synthesized using a one-pot method at ambient temperature. The red cabbage extract functioned as reducing agent and capping agent in the formation of the nanocrystallites products. XRD data confirmed the formation of CuI, and calculations showed that the sizes of the nanoparticles ranged from $85 \mathrm{~nm}$ to $92 \mathrm{~nm}$. The synthesis was environment-friendly since it solvent-free, straightforward, and one-pot. The synthesized $\mathrm{CuI}$ could be used as a colorimetric sensor for $\mathrm{Hg}^{0}$ vapor concentrations as low as $3.9 \mathrm{ppb}_{\mathrm{v}}$. CuI was immobilized on a cellulosic substrate using polystyrene as binder, and a smartphone camera was used to quantify the variation of the color upon exposure to $\mathrm{Hg}^{0}$ vapor. This sensing technique can be applied for monitoring exposure levels to $\mathrm{Hg}^{0}$ vapor, since the threshold exposure limit set by the World Health Organization is $6.1 \mathrm{ppb}_{\mathrm{v}}$ [27].

\section{CONFLICT OF INTEREST}

The authors declare no conflict of interest.

\section{ACKNOWLedgement}

A scholarship grant and research support fund was provided to one of the authors (ARMS) by the Accelerated Science and Technology Human Resource Development Program (ASTHRDP) of the Department of Science and Technology - Science Education Institute (DOST-SEI). 


\section{One-pot green synthesis of copper(I) iodide nanocrystallites}

\section{REFERENCES}

[1] Sharma B, Rabinal MK. Ambient synthesis and optoelectronic properties of copper iodide semiconductor nanoparticles. J Alloys Compd 2013; 556:198-202. DOI:10.1016/ j.jallcom.2012.12.120

[2] Ma Y, Gu M, Huang S, Liu X, Liu B, Ni C. Colloidal synthesis of uniform Cul nanoparticles and their size dependent optical properties. Mater Lett 2013; 100:166-169. DOI:10.1016/ j.matlet.2013.02.082

[3] Tavakoli F, Salavati-Niasari M, Mohandes F. Green synthesis of flower-like Cul microstructures composed of trigonal nanostructures using pomegranate juice. Mater Lett 2013; 100:133136. DOI:10.1016/j.matlet.2013.02.114

[4] Amalina MN, Azilawati Y, Rasheid NA, Rusop M. The properties of copper (I) iodide (Cul) thin films prepared by mister atomizer at different doping concentration. Procedia Eng 2013; 56:731-736. DOI:10.1016/j.proeng.2013.03.186

[5] Xu Y, Chen D, Jiao X, Ba L. PEG-assisted fabrication of single-crystalline Cul nanosheets: A general route to two-dimensional nanostructured materials. J Phys Chem C 2007; 111:6-9. DOI:10.1021/jp066649t

[6] Yang Y, Liu S, Kimura K. Synthesis of Welldispersed Cul Nanoparticles from an Available Solution Precursor. Chem Lett 2005; 34:11581159. DOI:10.1246/cl.2005.1158

[7] Vijayakumar A, Rajagopal R. Green Synthesis and Characterisation of Copper (I) Iodide nanoparticles using kidney bean seed extract and its anti-bacterial activity. Int J Sci Eng Res 2016; 7. http://www.ijser.org

[8] Shionoiri N, Sato T, Fujimori Y, Nakayama T, Nemoto M, Matsunaga T, Tanaka T. Investigation of the antiviral properties of copper iodide nanoparticles against feline calicivirus. $\mathrm{J}$ Biosci Bioeng 2012; 113:580-586. DOI:10.1016/ j.jbiosc.2011.12.006

[9] Salcedo ARM, Sevilla F. Colorimetric determination of mercury vapor using smartphone camera-based imaging. Instrum Sci Technol 2017; 46:450-462. DOI:10.1080/ 10739149.2017.1395745

[10] Yallouz AV, Cesar RG, Egler SG. Potential application of a semi-quantitative method for mercury determination in soils, sediments and gold mining residues. Environ Pollut 2008; 151:429-433. DOI:10.1016/j.envpol.2007.05.010
[11] Hu X, Yu JC, Gong J, Li Q. A facile surfaceetching route to thin films of metal iodides. Cryst Growth Des 2007; 7:262-267. DOI:10.1021/ cg060288p

[12] Prakash T. Influence Of Temperature On Physical Properties Of Copper(I) Iodide. Adv Mater Lett 2011; 2:131-135. DOI:10.5185/amlett.2011.1208

[13] Xu Y, Yang S, Zhang G, Sun Y, Gao D, Sun Y. Fabrication, characterization and optical property of Cul nanospheres. Mater Lett 2011; 65:16991702. DOI:10.1016/j.matlet.2011.03.016

[14] Zhang B, Xie A, Shen Y, Yang L, Huang Y, Lu J. Morphogenesis of Cul nanocrystals by a TSAAssisted photochemical route: Synthesis, optical properties, and growth mechanism. Eur J Inorg Chem 2009; 1376-1384. DOI:10.1002/ ejic. 200800966

[15] Shahbazi S, Afshar S. A facile, green, one pot synthesis of cuprous iodide nanoparticles using the mechanochemical method. Mater Lett 2014; 115:190-193. DOI:10.1016/j.matlet.2013.10.072

[16] Byranvand MM, Kharat ALIN. Triangular-Like Cuprous lodide Nanostructures: Green and Rapid Synthesis Using Sugar Beet Juice. Rom J Biochem 2014; 98:101-107.

[17] Byranvand MM, Kharat AN. One pot green synthesis of gold nanowires using pomegranate juice. Mater Lett 2014; 134:64-66. DOI:10.1016/ j.matlet.2014.07.046

[18] Mohammadinejad R, Karimi S, Iravani S, Varma RS. Plant-derived nanostructures: types and applications, Green Chem 2016; 18:20-52. DOI:10.1039/C5GC01403D

[19] Jungreis E. Spot test analysis: Clinical, environmental, forensic, and geochemical applications (2 ${ }^{\text {nd }}$ Ed.). (New York: Wiley, 1997).

[20] Dumarey R, Brown RJC, Corns WT, Brown AS, Stockwell PB. Elemental mercury vapour in air: The origins and validation of the "Dumarey equation" describing the mass concentration at saturation. Accredit Qual Assur 2010; 15:409_ 414. DOI:10.1007/s00769-010-0645-1

[21] Pandey SK, Kim K-H, Brown RJC. Measurement techniques for mercury species in ambient air. TrAC Trends Anal Chem 2011; 30:899-917. DOI:10.1016/j.trac.2011.01.017

[22] Tavakoli F, Salavati-Niasari M, Ghanbari D, Saberyan K, Hosseinpour-Mashkani SM. Application of glucose as a green capping agent and reductant to fabricate Cul micro/ nanostructures. Mater Res Bull 2014; 49:1420. DOI:10.1016/j.materresbull.2013.08.037 
[23] Mizgier $P$, Kucharska AZ, Sokó3-£etowska A, Kolniak-Ostek J, Kidoñ M, Fecka I. Characterization of phenolic compounds and antioxidant and anti-inflammatory properties of red cabbage and purple carrot extracts. J Funct Foods 2016; 21:133-146. DOI:10.1016/ j.jff.2015.12.004

[24] Pan J, Yang S, Li Y, Han L, Li X, Cui Y. Cul crystal growth in acetonitrile solvent by the cycleevaporation method. Cryst Growth Des 2009; 9:3825-3827. DOI:10.1021/cg900775a

[25] Xu Y, Chen D, Jiao X. PAM-assisted synthesis of single-crystalline Cul nanorods. Mater Lett 2009; 63:1859-1861. DOI:10.1016/j.matlet.2009.05.063
[26] Ferrante MJ, Mrazek RV, Brown RR. Hightemperature relative enthalpies and related thermodynamic properties of Cul (United States Bureau of Mines). (Pittsburgh, Pa: 1987). https:/ /nla.gov.au/nla.cat-vn3927582

[27] WHO. Recommended Health- Based Limits in Occupational Exposure to Heavy Metals (Report of WHO Study Group, World Health Organization). WHO Technical Report Series, No. 647. (Geneva, Switzerland: 1980). http:// apps.who.int/iris/bitstream/10665/41401/1/ WHO_TRS_647.pdf 